\title{
Políticas públicas y producción de espacios de vulnerabilidad. La construcción de la mujer-madre en el discurso político en España
}

\author{
Public policies and the production of vulnerability spaces. \\ The construction of the woman-mother in Spanish political discourse
}

SILVIA LÓPEZ-RODRÍGUEZ

Universidad Autónoma de Madrid

\section{Cómo citar/Citation}

López-Rodríguez, S. (2017). Políticas públicas y producción de espacios de vulnerabilidad. La construcción de la mujer-madre en el discurso político en España. Revista Española de Ciencia Política, 44, 97-120.Doi: https://doi.org/10.21 308/recp.44.04

\section{Resumen}

Este artículo explora la problematización de la maternidad y la producción del sujeto político «madre» en el actual discurso político en España. Concretamente, se interroga acerca de cómo las políticas públicas están implicadas en la producción diferencial de espacios de vulnerabilidad encarnada. Para ello se analiza el discurso político de dos áreas específicas: por un lado, la regulación de los derechos sexuales y reproductivos y, particularmente, del aborto, con especial interés en las implicaciones de las últimas reformas legislativas. Y por otro, la regulación del acceso diferencial a las técnicas de reproducción asistida en la sanidad pública, a partir sobre todo de la reforma normativa de 2014. Con ello, este artículo quiere contribuir a la literatura de análisis postestructural de políticas públicas, profundizando especialmente en los efectos del discurso político, tanto en la distribución de vulnerabilidad social y corporal como en la producción de subjetividad en clave de género.

Palabras clave: políticas públicas, género, espacio, discurso, maternidad, aborto.

\begin{abstract}
This article explores how the recent political discourse in Spain is problematizing motherhood and is producing "mothers" as political subjects. More in particular, the article is concerned about how public policies are involved in the production of differentiated spaces of bodily vulnerability. To do so, it analyses the policy discourse in two different areas: on the one hand, the regulation of sexual and reproductive rights and specifically abortion, paying
\end{abstract}


special attention to the latest legislative reforms; on the other hand, on the regulation of differentiated access to assisted reproduction techniques in public health services especially since the 2014 reforms. By doing so, this article aims at contributing to post-structural policy analysis literature, providing a deep insight into the effects of political discourse both on the distribution of social and bodily vulnerability and the production of gendered subjectivity.

Keywords: public policy, gender, space, discourse, motherhood, abortion.

[...they] often constructed that view around 'Mum', not as herself a living person engaged in the toils and troubles and pleasures of life, not actively engaged in her own and others' history, but a stable symbolic centre-functioning as an anchor for others.

Doreen Massey (1994): 180

Pregnancy does not belong to the woman herself. It is a state of the developing fetus, for which the woman is a container.

Iris M. Young (2005): 46

\section{INTRODUCIENDO UN ENFOQUE POSTESTRUCTURAL EN EL ANÁLISIS DE POLÍTICAS PÚBLICAS}

Este artículo tiene como objetivo analizar, de un lado, cómo nuestras políticas públicas actuales han problematizado la maternidad y, de otro, han producido un sujeto «madre» específico.

Para abordar el primer objetivo este trabajo se inspira en el enfoque de análisis de políticas públicas desarrollado en la obra de la analista Carol Bacchi (1999; 2009; Bacchi y Goodwin, 2016). Esta autora desafía los enfoques de análisis de políticas públicas que persiguen prioritariamente evaluar, cuantificar y medir los resultados de la implementación de las medidas políticas, de modo que afirma que dichas medidas tienen efectos mucho antes de su puesta en marcha (2017). Bacchi alega que las políticas públicas no abordan los problemas públicos sino que los conforman. De acuerdo con ello, este trabajo persigue analizar no cómo las políticas públicas se enfrentan a la maternidad como problema público, sino cómo estas dotan de significado -constituyen- la maternidad como problema público relevante. Con ello, la autora provee de un nuevo significado al concepto evaluación de políticas (policy «evaluation»" ${ }^{1}$ en la medida en que se propone evaluar cómo el discurso político y las políticas públicas son creadoras de significado (2009: XIV, 43).

El concepto de "problematización», de raíz foucaultiana (Bacchi, 2012; Bacchi y Bonham, 2014), sintetiza el interés de la autora por explorar cómo las políticas

1. Con comillas en el texto original. 
públicas constituyen, representan y dotan de significado los "problemas» que consideran relevantes (2009: 2, 263; 2012). El interés reside no en estudiar los problemas públicos que entran en la agenda política, sino en cuestionar la manera en que se problematizan diferentes situaciones señaladas como problemáticas. El concepto de problematización nos permite pensar en cómo las políticas públicas proporcionan mecanismos de enmarcado (framing mechanisms) que, de acuerdo a Bacchi, suponen formas de gobernanza específica (2009: 25 y ss.). Es decir, a través del estudio de las problematizaciones se pueden analizar efectos materiales que se plasmarán en cómo las personas vivimos nuestras vidas y cómo se conforma nuestra subjetividad (2012: 5).

Situar el foco de estudio en las problematizaciones que sostienen las políticas públicas no tiene un interés discursivo sino político: estudiando cómo se representan los problemas podemos rastrear repartos desiguales de poder. El interés no es pues meramente semiótico sino material: la manera en que los problemas se representan condiciona la vida cotidiana de las personas afectadas por la norma, las decisiones que pueden tomar, los espacios a los que pueden acceder, los derechos de los que son titulares, entre otros:

[...] la manera en que los problemas se constituyen (o conforman) conlleva todo tipo de implicaciones en relación a cómo vivimos nuestra vida cotidianamente. Es importante remarcar esto último porque la expresión representación del problema puede conducir a los lectores a pensar que actuamos solamente en el nivel de las ideas o las impresiones, cuando, en realidad, la manera en la que los problemas políticos son representados en las políticas públicas se trasladan a la experiencia real, vivida (Bacchi 2009: XVIII).

Las representaciones del problema o problematizaciones se constituyen en el discurso político (2009: 35), si bien el concepto de "discurso» que Bacchi abraza es deudor de la noción foucaultiana de "práctica discursiva" (Bacchi y Bonnham, 2014: 181). Tal como se acaba de exponer, el interés no es lingüístico sino político: el discurso de las políticas públicas produce un amplio rango de efectos que se materializan en experiencias cotidianas: es lo que Bacchi denomina lived effects, que en otros trabajos han sido traducidas como "experiencias encarnadas» (López Rodríguez, 2016). Esta aproximación permite explorar cómo las problematizaciones pueden provocar lo que Butler (2010) denomina "fenómenos políticamente inducidos», como la vulnerabilidad o la precariedad; o en palabras de Spade (2015), cómo las políticas y los programas distribuyen desigualmente oportunidades, seguridad y vulnerabilidad. También permite estudiar cuáles son los presupuestos normativos que están apuntalando las representaciones del problema o, en otros términos, qué se considera deseable socialmente. Como afirma Bacchi, en cada representación del problema se pueden identificar premisas conceptuales y normativas, sistemas de significados que conforman la lógica interna del relato y le da sentido (rationale) (2009: 4 y ss.). Esta misma idea es expuesta, partiendo asimismo de los presupuestos de Michel Foucault, por Dean Spade cuando señala cómo los proyectos deseables de Estado se articulan a 
través de políticas públicas y ello «implica proyectar ideas sobre la clase de vida que debe ser fomentada y la clase de vida que supone una amenaza y debe ser excluida, erradicada o extinguida" (2015: 123).

El hecho de que el foco dominante del análisis se sitúe no en el estudio de los problemas públicos sino en su producción discursiva sitúa al enfoque de Bacchi en el paradigma postestructural (2009: 34). Frente a los enfoques racionalistas o postpositivistas (discutidos en Guba, 1990; Bacchi, 1999; Goodwin, 2011; 2012), el interés de la propuesta de Bacchi reside en la puesta en cuestión de las propias políticas públicas como productoras de significado. El estudio de las problematizaciones permite asimismo analizar el poder desde diferentes dimensiones (2009: 37). En el ámbito de este trabajo es de interés analizar como uno de los efectos discursivos la manera en que el discurso político reparte diferencialmente seguridad e indemnidad, o vulnerabilidad y riesgo, particularmente en el ámbito corporal ${ }^{2}$.

Un enfoque como el aquí expuesto es especialmente atractivo para el estudio de la problematización de la maternidad en las políticas recientes. Así, este trabajo explora cómo la conformación de la maternidad implica la promoción de un modelo específico de feminidad y familia, que apuntala normas heteronormativas. Por otro lado, una perspectiva postestructural nos permite indagar en la vertiente ontológica de las políticas públicas, es decir, en la producción de subjetividad y sus consecuencias. El segundo objetivo de este trabajo consiste en estudiar cómo se ha conformado la mujer-madre como sujeto político normativo. Con ello, se pueden explorar no solo los procesos de inclusión y exclusión que toda categoría política conlleva sino también en un plano más amplio qué vidas se consideran significativas (Butler, 2003; 2006a; 2010) y qué vidas se consideran imposibles políticamente (Spade, 2015).

El artículo está dividido en cinco grandes apartados. En el próximo apartado se debate acerca del interés que reviste el estudio de la manera en que el discurso de nuestras políticas públicas está implicado en la producción de subjetividad y con qué consecuencias. En el apartado siguiente se introducen las implicaciones de estudiar cómo el discurso político distribuye vulnerabilidad desigualmente, hasta el punto de que sitúa a diferentes grupos de población en espacios diferenciales de vulnerabilidad corporal. A continuación, otro apartado aborda el estudio de la producción de la maternidad y de la mujer-madre en nuestro discurso político, argumentando que, a través de este estudio, se pueden detectar modelos deseables de feminidad y familia. En concreto, se analiza la regulación del acceso a la interrupción del embarazo y a la reproducción asistida. En el último apartado se debate las implicaciones del estudio de los espacios de vulnerabilidad corporal en lo que se refiere a las políticas relacionadas con la maternidad y la familia. Finalmente, se presentan algunas conclusiones.

2. Existe poca literatura en castellano que aborde el análisis de políticas públicas desde enfoques discursivos o postestrcuturales. No obstante, es ineludible citar el trabajo de Elin Peterson (2009, 2015) y de Alba Artiaga (2015) en el ámbito de estudio de las políticas de cuidados y la atención a la dependencia. 


\section{PROFUNDIZANDO EN LA DIMENSIÓN ONTOLÓGICA DEL ANÁLISIS DE POLÍTICAS PÚBLICAS}

El entendimiento del discurso político como práctica permite estudiar los efectos que se desprenden del relato de las políticas públicas. Por una parte, en este trabajo es de especial interés explorar cómo el discurso político produce subjetividad en clave de género. Por otra, esta investigación aspira a indagar acerca de cómo las políticas públicas condicionan las propias vidas, especialmente en una vertiente corporal.

Para abordar lo primero, este trabajo se alinea con la idea de que el discurso político produce subjetividad, es decir, el discurso político crea sujetos políticos a través de la creación de categorías, como las de «hombre», «mujer» o «madre» (Bacchi, 1996). Como han estudiado distintas autoras, el significado de estas categorías se constituye en el propio discurso y está sujeto a contestación y cambio (Hobson et al., 2002; Verloo y Lombardo, 2007; Butler, 2009). En el discurso político una categoría como la de «madre» no responde a una realidad física o biológica, sino a una decisión política de tipo normativo: la producción discursiva de la madre tendrá como consecuencia una madre normativa, deseable, y una madre rechazable (una "madre políticamente imposible», en la terminología de Spade) a la que se ocultará o se expulsará del debate.

Como afirma Tanesini en el caso de la categoría «mujer»: «[...] «mujer» es una noción normativa y, como tal, no es descriptiva de ningún hecho [...], el significado de «mujer» es materia de negociación, algo que puede ser desarrollado de muchos e imprevisibles modos» (1994: 212-213).

Siguiendo al analista Robert Chia (1996) la nota diferencial de la corriente postestructural es la distinción entre el ser (categorías incuestionadas: being) y el devenir (categorías en constante cuestionamiento y producción: becoming); de este modo, desde este enfoque prevalece una «ontología del devenir» (ontology of becoming), de la constitución y el cuestionamiento (citado en Bletsas, 2012: 41).

En el ámbito del análisis de políticas públicas, estudiar el proceso de subjetivación es importante en la medida en que nos permite analizar cómo las políticas públicas constituyen quiénes somos (Bacchi, 2009: 16 y ss.) y, especialmente, cómo se constituye a hombres y mujeres como sujetos políticos desiguales. Este proceso es denominado por la misma autora como gendering process (Bacchi, 2017). Es decir, se defiende una visión del análisis de políticas públicas cuyo foco se sitúe no en cómo dichas políticas afectan a hombres y mujeres sino en cómo las políticas públicas constituyen a hombres y mujeres, reproduciendo pautas de opresión y subordinación, o de emancipación. Siguiendo la línea del feminismo postestructural, se sostiene que hombres y mujeres no nos adscribimos a determinados rasgos disponibles de uno u otro género, sino que el género mismo es un proceso en constante replanteamiento y producción ${ }^{3}$,

3. En un plano teórico esta idea es expresada por los conceptos de performance (apropiación y teatralización de rasgos productores de género), "performatividad» (producción de ontología, de 
y como tal las propias categorías de "hombre» o «mujer» son objeto de discusión (por ejemplo, Butler 2009). Aplicando esta idea al análisis de políticas públicas podemos explorar cómo el discurso político crea relaciones de género (gendering) cuyos efectos podemos rastrear. Pero no solo eso; además se pueden explorar también procesos de reproducción de normas de clase, raza o heteronormatividad ${ }^{4}$. Para el propósito de este trabajo, el interés recae en el proceso de mothering ${ }^{5}$, es decir, cómo el discurso político construye la maternidad como proceso cargado de género, heteronormatividad y clase social. Y, a su vez, cómo se constituye un sujeto político "madre» que proyecta y defiende una madre normativa, situada en una posición de visibilidad y privilegio, y que invisibiliza a aquellas madres disidentes. Como sostendría Butler (2010), la cuestión no es qué mujer es madre sino qué mujer es reconocida como tal.

Esto supone abrir un debate ontológico en el seno del análisis de políticas públicas y situar un importante foco en la evaluación crítica del diseño de las mismas. Desde los enfoques contemporáneos del análisis feminista de políticas públicas se defiende dejar atrás el afán de incluir a los tradicionalmente excluidos (políticas de reconocimiento), para comenzar a cuestionar las categorías centrales del debate y diseñar políticas transformadoras (Squires, 1999; véase también Spade, 2015); así lo sintetiza Butler: «No es asunto de una simple inclusión de lo excluido dentro de una ontología establecida, sino de una insurrección al nivel de la ontología, una apertura crítica de las preguntas sobre qué es real, qué vidas son reales, cómo se podría rehacer la realidad» (2006a: 59).

En este trabajo el foco se sitúa no solo en explorar los procesos de inclusión y exclusión que la categoría «madre» produce, sino en cuestionar la constitución de esta categoría como productora de desigualdad. En último término, se analiza qué vidas cuentan o, reduciendo el foco, qué maternidades cuentan, las experiencias de qué madres son tomadas en cuenta y qué otras son constituidas como mujeres

subjetividad, por parte de las normas generizadas) y de «iterabilidad» (repetición continuada de esos rasgos), tan presentes en la literatura de Butler y a los que se ha dedicado tanto interés analítico. Un estudio muy reciente sobre todo ello desde la filosofía y el feminismo contemporáneo se recoge en el capítulo primero (acto primero) de De Mauro Rukovski (2015). El concepto de "performatividad» lo aborda asimismo con gran claridad el trabajo de Elvira Burgos (por ejemplo, 2012 y 2013).

4. Utilizamos este concepto de acuerdo a la formulación de Warner (1993) como el sistema social que supone la heterosexualidad como la única forma legítima de sexualidad y, por tanto, la única receptora de derechos de ciudadanía. Trujillo (2016) debate este concepto a la luz de la teoría feminista actual sobre el cuerpo.

5. Los conceptos de gender-ing o mother-ing se inspiran en los desarrollos más recientes de Carol Bacchi (2017). Aluden a la necesidad de estudiar cómo el discurso político produce relaciones de género o produce a las madres en tanto que sujetos políticos. El foco de análisis no se sitúa tanto en el estudio de categorías políticas concretas, como puede ser la de mujer o madre, sino en las relaciones de género o en la producción de las madres y de la maternidad como productos políticos y simbólicos. 
ajenas al marco de reconocibilidad de lo maternal. Como veremos después, la reforma que impide a las mujeres solas o a lesbianas en pareja acogerse a un tratamiento de fertilidad en la sanidad pública, en último término, las constituye como «madres imposibles» o como «mujeres con necesidades imposibles políticamente» (Spade, 2015).

La producción política de la madre supone asimismo una distribución de poder específica: las categorías no solo constituyen sujetos políticos desiguales, sino que también sitúan a estos sujetos en espacios de poder diferencial: espacios de riesgo o indemnidad; de vulnerabilidad o seguridad; de indemnidad o humillación. Espacios que condicionan las decisiones que se pueden tomar o las actuaciones que se deben evitar. Al estudiar esta producción de subjetividad en áreas de políticas públicas como la regulación de la interrupción del embarazo o el acceso a la sanidad pública es inevitable integrar la vertiente corporal en el análisis. En este trabajo se defiende que las políticas públicas distribuyen espacios diferenciales de vulnerabilidad, especialmente de vulnerabilidad corporal. El concepto de «espacio» tal y como lo entiende Doreen Massey nos permite pensar en estos términos, tal como se muestra a continuación.

\section{POLÍTICAS PÚBLICAS Y PRODUCCIÓN DE ESPACIOS DE VULNERABILIDAD}

En este trabajo se argumenta que la manera en que nuestras políticas públicas problematizan la maternidad va a dar lugar a la producción de un sujeto normativo madre, que puede en último término, provocar espacios de vulnerabilidad, riesgo o humillación, especialmente en un plano corporal, para aquellas mujeres que, aun siendo madres, no son reconocidas como tales por la norma. Es decir, la categoría política «madre» produce efectos subjetivadores que pueden redundar en la creación de una jerarquía entre las propias mujeres, de acuerdo a su adaptación o a su lejanía con respecto a la madre normativa. En otros términos, la norma sitúa a distintas mujeres en espacios diferenciales de poder: el acceso o el veto a la sanidad pública para interrumpir un embarazo de manera segura y gratuita, tomar la decisión de ser madres en un contexto distinto al de la pareja heterosexual, el reconocimiento de derechos en contextos de familias no tradicionales. A esto es a lo que Bacchi denomina los «efectos constitutivos del discurso político» (2017) o a lo que Spade alude como un tipo de poder, emanado de la ley, que distribuye oportunidades entre la población y al que, como tal, denomina "poder de ordenación de la población» (2015:119). Esta expresión de Spade nos devuelve a la importancia de pensar acerca de cómo el discurso político produce espacios coexistentes de poder desigual donde distintos grupos de población quedan situados diferencialmente. En este punto, la exploración del concepto de "espacio" parece esencial. Para ello nos servimos de la obra de Doreen Massey, cuyo concepto de «espacio» nos proporciona claves necesarias para los objetivos de esta investigación. 
En primer lugar, Massey entiende el espacio, no como una entidad meramente geográfica, sino política. En la extensa obra de la autora ${ }^{6}$, el concepto de espacio se estima relevante en la medida en que nos permite analizar las relaciones sociales que tienen lugar en su seno, especialmente las relaciones de género y clase (por ejemplo, 1993, 1994, 2012). $\mathrm{El}$ interés no reside, por tanto, en analizar la forma del espacio sino en estudiar su contenido (2008:101), es decir, la constelación de relaciones sociales que conforman el espacio y que sitúan a distintos grupos sociales en posiciones diferentes y desiguales. La clave pues no es estudiar dónde se sitúan las mujeres, sino cómo se constituyen las relaciones de género; la clave no es topográfica, es política: el objetivo último responde al estudio de relaciones de poder desigual. Es lo que Massey denomina como «geografía de relaciones sociales» (1993: 66) o "geografía de relaciones de poder» (1994: 22). Este concepto de espacio puede resultar útil para un tipo de análisis de políticas públicas como el aquí propuesto, en la medida en que se sostiene que el discurso político está implicado en la producción de espacios en los que pueden explorarse relaciones de poder desigual, analizando específicamente la dimensión de género. Este punto entronca con una segunda característica también presente en la obra de Massey: el espacio como producto del discurso.

En efecto, en una elocuente y reciente publicación, Massey argumenta cómo el espacio puede ser un producto discursivo: «El espacio es un producto social. Tanto el espacio, digamos, material como el espacio implícito en nuestros discursos e imaginaciones. Y, si es un producto social, entonces ha de ser también una responsabilidad política. Si es algo que producimos entonces importa cómo lo producimos» (2012: 9).

Este aspecto bien podría entrar en conversación con la elaboración de Carol Bacchi acerca del carácter constitutivo del discurso político, en un doble sentido. Por un lado, ambas autoras comparten un entendimiento del discurso como práctica constitutiva, como productora de efectos ligados a la distribución de desigualdad social. Es decir, el estudio del discurso importa en la medida en que se pueden analizar sus efectos, especialmente la manera en que distribuye poder, oportunidades, visibilidad, acceso a los recursos públicos y, como añadirá Massey, significado y simbolismo (1994: 3). Por otro lado, ambas autoras, sitúan su foco en una vertiente ontológica: cómo el discurso produce sujetos y, como tal, identidad. En su artículo más reciente, Bacchi (2017) estudia especialmente cómo el discurso político produce a hombres y mujeres como sujetos políticos desiguales; Massey, a su vez, admite cómo el estudio de los espacios discursivos y de las relaciones sociales en su seno permiten detectar no solo pautas de desigualdad, sino también efectos empoderantes o desempoderantes para las personas (2008: 100) e identidades cambiantes (1993: 5; 2008: 56) ${ }^{7}$. En síntesis, ambas autoras trazan un vín-

6. Aparte de la obra de la autora, no puede dejar de citarse el libro sobre la misma de Albet y Benach (2012), que compila distintos trabajos de Massey, un texto inédito, una entrevista y claves para su comprensión y lectura.

7. En este punto, Massey se apoya en gran medida en la obra de Chantal Mouffe en lo que concierne a sus ideas sobre la producción de subjetividad política. Massey conversa con Mouffe en este punto (2008: 10). 
culo entre el discurso político y la producción de subjetividad. O, en otros términos, entre el discurso político y la constitución de sujetos políticos desiguales, especialmente en clave de género.

En tercer lugar, Massey entiende que pueden coexistir distintos espacios donde el poder está desigualmente distribuido, donde se puede detectar «una compleja red de relaciones de dominación y subordinación, de solidaridad y cooperación ${ }^{8}$ (1994: 265). Es decir, se entiende que las personas se sitúan en espacios diferenciales de poder y que todos ellos son simultáneos, tal como esta cita clarifica:

Esta forma de conceptualizar el espacio, además, inherentemente implica la existencia en el mundo en el que vivimos de una multiplicidad de espacios simultáneos: espacios que se cruzan, se intersectan, se alinean unos con otros (...) las relaciones sociales del espacio se experimentan de manera diferente y se interpretan de manera variada por quienes quedan situados en diferentes posiciones (1994: 3$)^{9}$.

Es decir, la autora argumenta que distintos grupos sociales quedan situados en espacios diferenciales de poder, indemnidad y oportunidades, aunque compartan un mismo espacio geográfico. Así, por ejemplo, este planteamiento nos permite estudiar cómo existe una distribución de poder desigual entre las propias mujeres o, en otros términos, cómo el discurso político establece jerarquías entre los miembros del grupo social al que se dirigen sus acciones. De este modo, distintos grupos de mujeres pueden quedar situadas en espacios de vulnerabilidad diferenciales cuando el discurso político invisibiliza sus necesidades, margina sus voces o refuerza pautas de opresión previas. Tal es el ejemplo, en esta investigación, de las mujeres solas o en una pareja lesbiana a las que se niega el acceso a tratamientos de reproducción asistida en la sanidad pública: estas mujeres no solo quedan situadas en un espacio de vulnerabilidad y desigualdad de derechos, sino que se refuerza una pauta de opresión previa ligada al desafío de la norma heteronormativa de feminidad y familia.

La afirmación de que coexisten espacios de poder desigual donde, en este caso, quedan posicionados distintos grupos de mujeres, puede entenderse como una nueva manera de abordar la interseccionalidad (Crenshaw, 1991) ${ }^{10}$. En ese sentido, el foco no se situaría tanto en las situaciones de opresión que se derivan de la conjunción de distintos ejes de opresión (raza, género, clase social, estatus de migrante...), sino en cómo el discurso institucional condiciona la experiencia cotidiana de mujeres que sufren esas opresiones, situándolas en espacios donde su visibilidad, oportunidades, voz o acceso a

8. Si bien la conformación de redes de solidaridad no es abordada en este trabajo, es interesante al menos citar el concepto de "geografías de resistencia» que la autora maneja (2008: 103) y que entronca con el debate acerca de la capacidad de agencia de los sujetos en el contexto intelectual del análisis foucaultiano de discurso, del que sin duda esta investigación es también deudora. Una explicación imprescindible en este sentido es la de Dorthe Staunoes (2003).

9. Traducción propia del inglés original.

10. Véase sobre este mismo punto las conclusiones de López Rodríguez (2016). 
recursos es limitado o nulo. En el caso de los derechos sexuales y reproductivos, abordados en este trabajo, esos espacios están ligados al concepto de vulnerabilidad: como afirma Butler (2010), el discurso político puede ser el inductor de fenómenos como la precariedad o la vulnerabilidad, también en un plano corporal, que pueden derivar en una exposición diferencial a situaciones de humillación, riesgo, dolor o muerte prematura. El estudio de esta dimensión parece sin duda esencial en investigaciones desde el enfoque de género que aborden problemas públicos como la violencia de género, los derechos sexuales y reproductivos o el acceso a la salud.

El marco teórico hasta ahora expuesto permite formular las tres hipótesis que se van a desarrollar a continuación en el estudio de caso. En primer lugar, este trabajo sostiene que el discurso de las políticas públicas produce espacios de vulnerabilidad, es decir, espacios donde el poder y las oportunidades quedan desigualmente repartidas. El foco de este trabajo se sitúa en un tipo de vulnerabilidad específica: la vulnerabilidad corporal. Con ello, se pretende explorar cómo el discurso de nuestras políticas públicas en el ámbito de los derechos sexuales y reproductivos, la maternidad y la familia sitúan a distintos grupos de mujeres en espacios diferenciales de vulnerabilidad corporal. En segundo lugar, se argumenta que la manera en que el discurso institucional problematiza la maternidad tiende a reforzar entendimientos de la maternidad y la familia que refuerzan la creación de espacios de desigualdad entre las propias mujeres y puede además consolidar y promover una pauta heteronormativa de comportamiento. Unido a ello, la tercera hipótesis sostiene que el discurso político tiene efectos subjetivadores y, como tal, produce una madre normativa. A partir del estudio de esta categoría política se pueden explorar asimismo no solo procesos de inclusión y exclusión de la norma, sino también la manera en que se legitiman determinadas vidas, experiencias y valores sociales considerados valiosos, al tiempo que se rechazan o invisibilizan vidas, experiencias o valores considerados imposibles políticamente (Spade, 2015). Una reflexión con profundas implicaciones en el diseño y evaluación de políticas públicas. Todo ello se desarrollará en el estudio de expuesto en los siguientes epígrafes.

\section{ESTUDIO DE CASO: LA MATERNIDAD COMO CONSTRUCCIÓN POLÍTICA}

Para desarrollar las hipótesis que se acaban de exponer y de acuerdo al marco teórico expuesto, este trabajo sitúa el foco en el análisis del discurso político de la décima legislatura en España (2011-2015), especialmente en lo que concierne a la regulación de la maternidad y el parentesco. La elección de esta legislatura responde a la presencia de un profundo cambio en la manera en que se problematizan las relaciones de género y la maternidad con respecto a la etapa anterior.

Esta investigación apuesta por una metodología de análisis postestructural de políticas públicas inspirada en el trabajo de Carol Bacchi (1996; 1999; 2009; Bacchi y Goodwin, 2016). Como se ha visto, esta autora entiende que las políticas públicas pueden estudiarse como discursos políticos que tienen un impacto en la vivencia 
cotidiana de las personas (2009: XVIII) y especialmente en los cuerpos a los que la norma concierne (1999: 46). El estudio de las políticas como discurso nos permitirá estudiar la problematización de la maternidad, la constitución del sujeto madre y el análisis de los espacios de vulnerabilidad consiguientes. Así esta investigación tratará de responder a preguntas acerca de cómo se constituyen la feminidad, la maternidad y la familia en nuestro discurso político, y cómo se constituyen -y con qué efectos- los sujetos políticos «mujer» y «madre». Además, de acuerdo con las propuestas de Butler y Massey recién expuestas, la investigación explora cómo el discurso político puede producir situaciones de vulnerabilidad y, particularmente, espacios de vulnerabilidad diferencial para determinadas mujeres.

Para articular el estudio del discurso político de interés para este trabajo, se han seleccionado un conjunto de textos políticos considerados relevantes para los objetivos de esta investigación (leyes aprobadas durante la legislatura, actas parlamentarias, programas electorales). A estos documentos objeto de análisis Carol Bacchi los denomina practical texts (2009: 34, 249) ${ }^{11}$. De acuerdo a la metodología desarrollada por esta autora y siguiendo el contenido que Michel Foucault le da a este concepto, estudiar nuestras políticas públicas y, en general, nuestros discursos políticos como «textos prácticos» implica entender dichos discursos como prácticas políticas: el discurso tiene un carácter prescriptivo, aspira a conformar el marco de comportamiento de nuestra conducta cotidiana (Bacchi, 2009: 34). El trabajo con estos textos nos permite interrogarnos acerca de los objetivos de esta investigación: no solo rastrear las problematizaciones alrededor de la maternidad, sino explorar la vertiente ontológica del discurso político. Y en último término interrogarnos acerca de los espacios de vulnerabilidad que todo ello induce.

El siguiente apartado explora las hipótesis expuestas en el caso de la regulación de los derechos sexuales y reproductivos y, particularmente, del acceso a la interrupción del embarazo. A continuación, se estudia la regulación del acceso a la reproducción asistida en la sanidad pública, explorando así la regulación del parentesco y la filiación ${ }^{12}$.

\section{La constitución de la mujer-madre}

La primera regulación de la interrupción del embarazo en el período democrático actual $^{13}$ se produce con la reforma del Código Penal de 1983, reforma impugnada ante

11. Estos «textos prácticos» aparecen reseñados y analizados en los epígrafes siguientes.

12. Para seguir profundizando en el debate acerca de modelos de familia y maternidad que desafían la norma heteronormativa dominante, son de interés Gras Velázquez (2014), Borrás (2014) y Llopis (2015). Agradezco a una de las personas que ha evaluado este artículo que me haya llamado la atención sobre estas fuentes.

13. Durante la Segunda República, en Cataluña sale adelante un decreto (aprobado el 25 de diciembre de 1936 y publicado el 9 de enero de 1937) que permite la interrupción del embarazo por las denominadas «razones éticas», que se refieren al «deseo consciente de limitar voluntariamente la natalidad». Federica Montseny (ministra de Sanidad y Asistencia Pública) intentó 
el Tribunal Constitucional, que en 1985 declara, no obstante, su carácter constitucional, entrando en vigor ese mismo año ${ }^{14}$. Esta reforma permite la interrupción del embarazo ante la concurrencia de alguno de estos supuestos: grave peligro para la vida o la salud física o psíquica de la embarazada, embarazo fruto de un delito de violación mediando denuncia de la víctima y no más tarde de la duodécima semana o que se detecten en el feto "graves taras físicas o psíquicas» no más tarde de la semana vigesimosegunda del embarazo. En 1995 se aprueba una nueva ley contemplando un cuarto supuesto relacionado con la existencia de un "conflicto personal, familiar o social para la mujer» (art. 1.2.), pero la convocatoria de elecciones anticipadas impide finalizar su tramitación ${ }^{15}$.

Será en el año 2010 cuando se pase de un sistema de supuestos a la legalización de la interrupción del embarazo en España en el marco de un sistema de plazos ${ }^{16}$. Esta ley, aprobada en la etapa final de la segunda legislatura de Rodríguez Zapatero, permite analizar tanto la manera en que se problematiza la maternidad como la forma en que se constituye el sujeto «madre». La Ley de 2010 sitúa el tratamiento de la maternidad en un marco más amplio y previo: el marco del desarrollo de la sexualidad y de los derechos sexuales y reproductivos, ligados a derechos fundamentales que es preciso proteger: la dignidad de la persona, el libre desarrollo de su personalidad, la intimidad personal y familiar, y la integridad física y moral (preámbulo I). En este marco se introduce una narrativa que atraviesa el texto de la ley: cómo las relaciones de género van a condicionar el pleno desarrollo de esos derechos. La Ley constituye dos sujetos políticos en relación: hombres y mujeres, para quienes -se argumenta- la parentalidad y el desarrollo de la sexualidad tienen significados e implicaciones distintas. Así, el texto admite que «la protección de este ámbito de autonomía personal tiene una singular significación para las mujeres para quienes el embarazo y la maternidad son hechos que afectan profundamente sus vidas» (preámbulo I). De ahí que la Ley persiga reconocer el «derecho a una maternidad libremente decidida" (preámbulo II) y a trabajar para impedir embarazos indeseados a través del fomento de relaciones de género más igualitarias. En este sentido, la Ley prevé una «educación sanitaria integral y con perspectiva de género sobre salud sexual y reproductiva» (art. 5.1. e), el fomento de «relaciones de igualdad y respeto mutuo entre hombres y mujeres en el ámbito de la salud sexual» (art. 5. 2. a), la «corresponsabilidad en la conducta sexual» (art. 5.2. b) y la promoción de una «visión de la sexualidad en términos de igualdad y corresponsabilidad entre hombres y mujeres,

ampliar la aplicación de ese decreto al resto de áreas republicanas, pero el triunfo del golpe militar acabó con la derogación de esta norma.

14. Ley Orgánica 9/1985, de 5 de julio de reforma del artículo 417 bis del Código Penal. La sentencia que declara la constitucionalidad de la norma, rescatada en los debates de la ley posterior de 2010 y, especialmente, en el debate acerca del anteproyecto de reforma de Ruiz-Gallardón, es la Sentencia 53/1985, de 11 de abril.

15. Ley Orgánica sobre Regulación de la Interrupción Voluntaria del Embarazo, BOE, 25 de julio de 1995; se aprueba el 14 de diciembre de ese mismo año en sede parlamentaria.

16. Ley Orgánica $2 / 2010$, de 3 de marzo, de salud sexual y reproductiva y de la interrupción voluntaria del embarazo. 
con especial atención a la prevención de la violencia de género, agresiones y abusos sexuales» (art. 9 a). La ley, por tanto, articula un discurso con dos sujetos políticos desiguales: hombres y mujeres. Y entiende que los embarazos indeseados no son el problema, sino, en ocasiones, el resultado de relaciones de género desiguales sobre las que es necesario intervenir. La problematización de la maternidad va ligada a normas relacionadas con la autonomía de la mujer (preámbulo I y II) y a su libre decisión (preámbulo II; art. 3.2.), en el marco de derechos fundamentales como la libertad, la intimidad y la autonomía personal (art. 3.1.).

El discurso institucional en materia de interrupción del embarazo en España sufre un vuelco con el comienzo de la décima legislatura (2011-2015) y el nuevo Gobierno del Partido Popular. Éste impulsa la derogación de la Ley de 2010 y la aprobación de un nuevo texto normativo, impulsado por el entonces ministro de Justicia, Alberto Ruiz-Gallardón. El 20 de diciembre de 2013 se aprueba el Anteproyecto de Ley Orgánica para la Protección del Concebido No Nacido y de los Derechos de la Mujer Embarazada, que retoma un sistema de supuestos más restringido que el contemplado en la reforma del Código Penal de $1983^{17}$. En este texto desaparece la narrativa de la desigualdad de género; el proceso de subjetivación que se deriva del Anteproyecto constituye a dos sujetos enfrentados: la madre y el concebido no-nacido. Con respecto a la primera, el texto normativo consolida un nuevo sujeto político, la mujer-madre: desaparece del discurso la mujer que, en el marco de su autonomía personal decide sobre sus derechos sexuales y reproductivos; desaparece asimismo la mujer embarazada y se constituye una mujer-madre normativa. Por un lado, una madre objeto de una «violencia estructural» ${ }^{18}$, de una difusa coacción social que le puede llevar a interrumpir su embarazo «sin su consentimiento o bajo intimidación o engaño» (exposición de motivos del Anteproyecto IV). Por otro lado, la madre que se enfrenta a la interrupción de su embarazo de acuerdo al sistema de supuestos establecidos en la norma se constituye como víctima de una «situación de grave conflicto personal» (exposición de motivos II) que se enfrenta a "un drama, un conflicto insuperable»" ${ }^{19}$ de ahí que se suprima todo reproche penal hacia ell ${ }^{20}$. Frente a la madre, se constituye un sujeto político nuevo en el discurso institucional español en esta materia: «el concebido no nacido», el más débil y cuyos derechos es necesario salvaguardar, especialmente, el derecho a la vida: «Sea usted auténticamente progresista y defienda de verdad a los más débiles y piense usted que, en este caso, el más débil, el que necesita la protección es el concebido $»^{21}$.

17. Más sobre ello en López Rodríguez (2016). El supuesto de interrupción del embarazo por graves anomalías fetales, contemplado en 1983, queda en entredicho en el Anteproyecto de 2013 si la salud de la madre no está en riesgo.

18. Alberto Ruiz-Gallardón. Diario de Sesiones, núm. 16 de 7 de marzo de 2012, p. 10.

19. Alberto Ruiz-Gallardón. Diario de Sesiones, núm. 172 de 22 de enero de 2014, p. 86.

20. Alberto Ruiz-Gallardón. Diario de Sesiones, núm. 113 de 22 de mayo de 2013, p. 28.

21. Alberto Ruiz-Gallardón. Diario de Sesiones, núm. 116 de 29 de mayo de 2013, p. 20. 
Si en la Ley de 2010 se constituye a hombres y mujeres como sujetos políticos desiguales, en este Anteproyecto la relación de desigualdad se constituye entre la madre y el concebido no nacido, el más débil en la relación y, por lo tanto, aquel a quien hay que proteger en primer término. Si en la Ley de 2010 la estrategia que predomina es la de la prevención de embarazos indeseados, en el caso de este Anteproyecto la estrategia dominante tiene que ver con la prevención de interrupciones del embarazo. Y ello porque se problematiza la maternidad como un valor fundamental de la feminidad ${ }^{22}$, de modo que las categorías políticas de mujer y de madre se constituyen como una sola. La maternidad se conforma no como un fenómeno biológico sino como un proyecto político encarnado en el cuerpo femenino, que proyecta un modelo deseabilidad social de feminidad y familia. El foco de la problematización se sitúa en el concebido no nacido y en la promoción misma de los nacimientos, lo que supone dejar sin debatir las condiciones que hacen una vida posible: la promoción de los nacimientos no conlleva al tiempo la promoción de una vida sostenible ${ }^{23}$. Releyendo a Butler para este caso específico, la cuestión no sería si el concebido no nacido es el más débil o si es un ser vivo sino si «las condiciones sociales de su persistencia y prosperidad son o no posibles» (2010: 38-39). En la medida en que la «mujer» desaparece del discurso es imposible debatir acerca de las condiciones de vida en las que se desenvuelve para la toma de sus decisiones.

La fuerte contestación social del movimiento feminista ${ }^{24} \mathrm{y}$ las divisiones internas del Gobierno en torno a este Anteproyecto llevaron a que el presidente del Gobierno, Mariano Rajoy, lo retirara el 23 de septiembre de 2014, lo que desencadenó la dimisión del ministro Ruiz-Gallardón, su abandono del Partido Popular y de la actividad política.

No obstante, el Gobierno Popular se comprometió a modificar las condiciones en las que las jóvenes de diceiséis y diecisiete años pueden acceder a la interrupción del embarazo. Según la Ley de 2010, las menores han de informar a sus progenitores o tutores legales, a no ser que la menor alegue que esta información le supondrá un conflicto grave en el entorno familiar o manifiesto peligro de violencia (art. 13). Con la aprobación de la Ley Orgánica $11 / 2015^{25}$, se exige a las menores un «consentimiento expreso» para poder acceder a la interrupción de su embarazo. Esta «práctica divisoria» (Bacchi, 2009: 16-17) entre mujeres mayores y menores de edad ha reforzado la situación de vulnerabilidad de aquellas menores de edad con situaciones de partida más desfavorables, situándolas en un espacio de vulnerabilidad diferencial, como se mostrará más ampliamente en el apartado

22. Alberto Ruiz-Gallardón. Diario de Sesiones, núm. 16 de 7 de marzo de 2012, p. 10.

23. Idea que es reforzada en el programa electoral presentado por el Partido Popular a las elecciones de 2015 y 2016, al situar el foco en la promoción de la maternidad, a través del Plan de Apoyo a la Familia y el Plan de Apoyo a la Maternidad (págs. 93-94).

24. Especialmente la multitudinaria manifestación en Madrid del 1 de febrero de 2014, bautizada como «El tren de la libertad», impulsada por los colectivos feministas Las Comadres (Asturias).

25. Ley Orgánica 11/2015, de 21 de septiembre, para reforzar la protección de las menores y mujeres con capacidad modificada judicialmente en la interrupción voluntaria del embarazo. 
final. Particularmente grave ha sido esta situación en la Comunidad de Madrid, pues exigía a las menores el consentimiento expreso de ambos progenitores, un doble consentimiento inaccesible para menores en familias monoparentales o familias de padres ausentes o violentos. Este caso ilustra, por tanto, cómo la norma puede reforzar situaciones de vulnerabilidad previas, particularmente de vulnerabilidad corporal, pues al carecer de este doble consentimiento el riesgo en el embarazo puede aumentar o la tardanza en conseguir el trámite puede suponer que el embarazo avance hasta superar los plazos previstos en la ley. El doble consentimiento está proyectando una idea de familia estructurada, tradicional, heteronormativa, que excluye situaciones de particular vulnerabilidad, como las que citaba el representante de la Asociación de Clínicas Acreditadas para la Interrupción del Embarazo en la sede de la Asamblea de Madrid: «Menor que vive con la madre y que el padre desapareció tras su nacimiento; menor con padre en prisión; menor extranjera que vive con la madre y cuyo padre vive en Bolivia en paradero desconocido; menor española que vive con la madre y que el padre trabaja en el extranjero» ${ }^{26}$.

Este ejemplo ilustra cómo la norma refuerza pautas de desigualdad de partida, repartiendo diferencialmente oportunidades, seguridad y vulnerabilidad (Spade, 2015: 123, 127) y sitúa a aquellas jóvenes en situaciones más precarias de inicio en espacios de vulnerabilidad específicos. Esta vulnerabilidad tiene inevitablemente una vertiente encarnada: la dificultad de acceso al doble consentimiento sitúa la vulnerabilidad de su cuerpo en el centro, pudiendo enfrentarse eventualmente a situaciones no solo de mayor riesgo para su salud y su vida, sino también a una humillación y sufrimiento diferencial.

\section{La constitución de madres imposibles}

La regulación del parentesco y la filiación constituyen asimismo áreas privilegiadas para estudiar la manera en que se constituye el sujeto político madre y se problematiza la maternidad como institución social. En el marco de la décima legislatura, foco de este trabajo, se impulsa desde el Ministerio de Sanidad una reforma normativa en materia de reproducción asistida que resulta en el veto a estos tratamientos en la sanidad pública a mujeres en parejas lesbianas o solas. Así se afirma en la orden ministerial aprobada en el año $2014^{27}$ : «Los tratamientos de reproducción humana asistida (RHA) se realizarán con fin terapéutico o preventivo y en determinadas situaciones especiales» ${ }^{28}$

26. José Antonio Bosch. Diario de Sesiones de la Asamblea de Madrid, núm. 217, de 17 de mayo de 2016, p. 14. En el mismo sentido, Clara Serra, Diario de Sesiones de la Asamblea de Madrid, núm. 226, de 2 de junio de 2016, pp. 120-121.

27. Orden SSI/ 2065/2014, de 31 de octubre, por la que se modifican los anexos I, II y III del Real Decreto 1030/2006, de 15 de septiembre, por el que se establece la cartera de servicios comunes del Sistema Nacional de Salud y el procedimiento para su actualización.

28. Pág. 91373, de la orden ministerial citada en la nota al pie anterior. 
Es decir, los procedimientos de reproducción asistida quedan reservados a aquellas parejas heterosexuales que, por alguna condición médica, no puedan concebir sin procedimientos médicos. Y como afirmó la entonces ministra de Sanidad, Ana Mato: «La falta de varón no es un problema médico ${ }^{29}$. $\mathrm{O}$ como reconoció la portavoz popular Carmen Quintanilla: «La reproducción asistida es un privilegio [de parejas heterosexuales] no un derecho [de todas las mujeres/ parejas] ${ }^{30}$.

Esta norma introduce un discurso que constituye las relaciones de parentesco y filiación como heterosexuales de antemano, de modo que la heterosexualidad se instituye como una estructura fundamentadora del parentesco (Butler, 2006b: 178). Este discurso ejemplifica asimismo aquello que Monique Wittig (2005) denominó la «mente heterosexual»: el presupuesto de partida de que las familias, las relaciones sociales, las ideas sobre el amor son implícitamente heterosexuales (Trujillo 2016: 61-62). El discurso político constituye la relación heterosexual y la familia heteronormativa en una institución política hegemónica, que no solamente excluye otras formas de parentesco y filiación sino que convierte a determinadas vidas y necesidades como imposibles políticamente.

En efecto, las mujeres en una pareja lesbiana o solas con cualquier orientación del deseo sexual se constituyen como "madres imposibles», usando la terminología de Spade (2015: 21). No solo es interesante observar cómo esta norma y el discurso político que la acompaña distribuye diferencialmente oportunidades, visibilidad y recursos sino también estudiar cómo el discurso crea ontología; el análisis no se centra exclusivamente en un mero proceso de exclusión de la norma de determinadas mujeres sino en la ontología misma y sus efectos: a qué madres legitima la norma, qué mujeres se entienden como madres imposibles. De nuevo, la maternidad se problematiza como un producto simbólico, una institución política al servicio de determinados intereses y normas sociales vinculados a la reproducción de la heteronormatividad y encarnados diferencialmente en el cuerpo femenino.

Butler (2002) se referirá en esta misma línea a los «cuerpos abyectos», cuerpos que son instituidos discursivamente como no significativos (Burgos, 2012: 117). Así, el discurso político produce cuerpos y necesidades ininteligibles políticamente, ante lo que no cabe una mera estrategia de cuestionamiento de dicha exclusión sino, más bien, de debate acerca de cómo la producción misma de categorías políticas da lugar a sujetos que son arrojados fuera de las posibilidades de lo humano. La lógica no es de mera jerarquía entre los cuerpos sino de cómo el discurso institucional produce cuerpos «que no importan»: «Lo abyecto es resultado de un ejercicio discursivo del poder, del mismo ejercicio que establece el dominio de lo que es un cuerpo como tal, de realidad ontológica dada» (Burgos, 2013: 207).

Ante ello, la estrategia no puede ser diseñar políticas más inclusivas, sino cuestionar las políticas mismas (de ahí, el carácter deconstructivo) para pensarlas de nuevo en

29. Comparecencia ante la prensa, 23-07-2013.

30. Entrevista en eldiario.es, 17-12-2013. 
direcciones más humanizadoras, un aspecto nada despreciable en el diseño de políticas públicas: «El problema no es meramente cómo incluir a más personas dentro de las normas [políticas] ya existentes, sino considerar cómo las normas ya existentes asignan reconocimiento de manera diferencial» (Butler, 2010: 20).

El cuerpo de las mujeres en parejas lesbianas o solas no es reconocido como un cuerpo maternal, así como las familias de las que forman parte son producidas fuera de lo políticamente comprensible. Ello da lugar al refuerzo de pautas heternormativas de comportamiento y a la conversión de la heterosexualidad en una estructura hegemónica, de sentido común, en la sociedad. La familia heterosexual deja de ser en cierto modo un modelo de parentesco para devenir un proyecto político sustentador de lo que Butler denomina la «cultura monolítica» (2006b: 180).

La Orden Ministerial ha provocado una enorme contestación por parte de las comunidades autónomas, entidades encargadas de desarrollar la norma, al punto de que consejeros y consejeras como los de Cantabria, Comunidad Valenciana, Andalucía o Euskadi han hecho pública su voluntad de mantener los tratamientos de reproducción asistida de manera independiente a la orientación del deseo sexual o al modelo de familia. No obstante, otras regiones -como la Comunidad de Madrid-, que sí están implementando la norma, sitúan a determinadas mujeres en espacios específicos de vulnerabilidad encarnada, por ejemplo: si el tratamiento al que estaban siendo sometidas se interrumpe o si han de recurrir a centros privados para llevarlos a cabo. De dichos espacios trata el apartado siguiente.

\section{LA PRODUCCIÓN POLÍTICA DE ESPACIOS DE VULNERABILIDAD CORPORAL}

Desde el análisis de políticas públicas de corte postestructural, Carol Bacchi ha mostrado la importancia de indagar acerca de los efectos subjetivadores del discurso político, es decir, cómo el discurso nos constituye como sujetos políticos (2009: 39 y ss.) y, específicamente, cómo produce a hombres y mujeres como sujetos políticos desiguales. El foco de análisis se sitúa en la producción misma de relaciones de género por parte de nuestras políticas (gender-ing, 2017). En la misma línea, Judith Butler muestra una preocupación por analizar las categorías políticas que tienen efectos identitarios, no con el ánimo de estudiar procesos de inclusión y exclusión, sino para interrogarse acerca de las vidas que se consideran legítimas y significativas. La autora reconoce en su obra la violencia misma del acto de nombrar y el carácter necesariamente excluyente de todo proceso de categorización (2009: 276-277); no obstante, el estudio de categorías como las de «mujer» $\mathrm{o}$ «madre» tiene interés para ella -sobre todo en el discurso de las políticas públicas- porque el proceso de subjetivación, y especialmente sus efectos, va ligado a la posibilidad misma del desarrollo de las ilusiones, los afectos, los proyectos y, en definitiva, de una vida en libertad (Burgos, 2013). Así, afirma Butler: «Si proponemos leyes que están específicamente pensadas para proteger a las mujeres, para proteger a ciertas identidades del daño, entonces surgen algunas dificultades [...]. Si hay leyes para 
proteger exclusivamente a las mujeres, entonces creo que debemos preguntar, ¿quién es una mujer, quién cuenta como una mujer?» (Burgos, 2008: 413).

Aquellas que, aun siendo mujeres, quedan fuera del marco de reconocibilidad de lo femenino o lo maternal, quedan situadas en un espacio de vulnerabilidad que ha sido producido políticamente. El concepto de «espacio» que Doreen Massey propone nos ayuda a pensar acerca de cómo las políticas públicas constituyen espacios diferenciales de poder donde distintas mujeres quedan desigualmente situadas. Este concepto de espacio no tiene como ánimo realizar una topografía de la localización de las mujeres, sino que más bien se propone estudiar distribuciones desiguales de poder, recursos, voz, visibilidad, oportunidades entre las mujeres, que refuerzan en ocasiones pautas de desigualdad de partida (marcadas por el género, la situación familiar, la situación socioeconómica, la orientación del deseo sexual). La formulación de Massey de su concepto de espacio muestra una enorme utilidad para este trabajo en la medida en que proporciona un instrumento para estudiar situaciones de vulnerabilidad diferencial y, de manera particular, de vulnerabilidad corporal que son provocadas por el discurso político.

De acuerdo con los resultados de esta investigación, en lo que concierne a las condiciones de acceso a la interrupción del embarazo, la reforma del año 2015, que exige a las jóvenes de diceciséis y diecisiete años el consentimiento expreso de sus padres o tutores legales como requisito previo para abortar en la sanidad pública, ha situado a estas jóvenes en un espacio de vulnerabilidad específico, al tiempo que ha reforzado pautas de vulnerabilidad previas. El caso específico de la Comunidad de Madrid, donde se exige un doble consentimiento, ha mostrado cómo jóvenes con situaciones de partida más desfavorables (padres presos, ausentes o violentos) se sitúan en un espacio de mayor vulnerabilidad y de menores oportunidades y seguridad, en el marco de un contexto corporal específico: un embarazo indeseado. Es más, como señala Spade (2015) es necesario analizar aspectos inmateriales que tienen una clara vertiente encarnada: cómo la norma pública puede generar humillación, angustia o sufrimiento, en lo que él denomina «violencia administrativa». Sirva como ejemplo el de una joven menor de edad que desea interrumpir su embarazo y a la que se le exige también el consentimiento de su padre, denunciado por su madre por violencia de género ${ }^{31}$. Al mismo tiempo, este ejemplo nos permite mostrar cómo la norma reproduce y legitima un modelo determinado de familia (tradicional, estructurada, heteronormativa) que sitúa en un espacio de vulnerabilidad a aquellas otras familias que no se adaptan al modelo convertido en normativo ${ }^{32}$.

El caso de las restricciones en el acceso a la reproducción asistida en la sanidad pública nos muestra cómo las mujeres lesbianas en pareja o solas de cualquier orientación del deseo sexual quedan situadas en un espacio de vulnerabilidad que también tiene a su

31. Clara Serra. Diario de Sesiones de la Asamblea de Madrid, núm. 226, pp. 120-121.

32. Un espacio de vulnerabilidad adicional sería aquel en el que quedan situadas mujeres indocumentadas a las que se les retira la tarjeta sanitaria (Real Decreto-ley 16/2012, de 20 de abril) y, como tal, quedan expulsadas de los servicios públicos de salud para interrumpir un embarazo indeseado. Este caso se trata en Lopez Rodríguez (2016). 
cuerpo en el centro: un cuerpo maternal ininteligible, abyecto (Butler, 2002) y de necesidades políticamente imposibles (Spade, 2015). El refuerzo de un modelo heteronormativo de comportamiento deja sin reconocer determinadas vidas, experiencias y familias, que son ajenas al debate político, al sentido común promovido por el discurso hegemónico. Estas «madres imposibles» quedan ajenas al marco de reconocimiento de lo femenino y de lo maternal, quedando expuestas, como en el caso anterior, a situaciones de humillación e inseguridad diferenciales: tal es el caso de parejas cuyo tratamiento se interrumpe o se ve en peligro una vez la norma es aprobada, como muestra esta entrevistada: «Las restricciones en los tratamientos de reproducción asistida en la sanidad pública nos crearon a mi pareja y a mí una situación de gran tensión en pleno proceso de embarazo porque no sabíamos si nos iban a dejar fuera del programa ${ }^{33}$.

La Orden Ministerial de 2014 reproduce asimismo lo que Wittig (2005) teorizó como el «pensamiento heterosexual»: el presupuesto de que la norma de comportamiento humana (y, como tal, su regulación) es heterosexual.

El estudio de cómo nuestras normas producen espacios de vulnerabilidad corporal diferenciales se muestra de enorme importancia para el análisis y la elaboración de nuestras políticas, especialmente en lo que concierne a su diseño. Butler interroga al análisis de políticas públicas así: «¿Qué nuevas normas son posibles y cómo son producidas? ¿Qué podría hacerse para producir una serie más igualitaria de las condiciones de reconocibilidad? En otras palabras, ¿qué podría hacerse para cambiar los términos mismos de la reconocibilidad con el fin de producir unos resultados más radicalmente democráticos?» (2010: 20).

Si sostenemos que nuestras políticas públicas tienen la capacidad de producir espacios de vulnerabilidad corporal, repartos diferenciales de poder, recursos y oportunidades, sujetos políticos a los que se reconoce diferencialmente, también podemos alegar, en sentido contrario, que desde las políticas públicas se pueden promover espacios emancipadores, así como identidades que reconozcan la pluralidad de dimensiones de la elección humana. Con ello, sirva este trabajo para visibilizar el vínculo que existe entre nuestras políticas públicas y los espacios de posibilidad en los que quedamos situados: espacios en los que necesariamente se desarrollan nuestras decisiones, deseos y proyectos. Pensar el análisis de políticas públicas desde esta perspectiva, no solo nos invita a pensar en los pilares del reconocimiento sino, como asume Butler, en los de la democracia misma.

\section{CONCLUSIONES}

Esta investigación se ha interrogado acerca del modo en que el discurso político en España ha problematizado la «maternidad» no como un fenómeno biológico, sino

33. Entrevista a Alba García, recogida en El Diario.es el 24-10-2015 (disponible en: http://www. eldiario.es/cv/convirtio-angustioso-reproduccion-asistida-lesbianas_0_444455800.html) 
como un producto simbólico y político, que resulta en la distribución diferencial de oportunidades y recursos entre las mujeres y las familias. Al mismo tiempo, este trabajo indaga sobre el poder del discurso institucional en la creación de ontología; particularmente, se ha expuesto la manera en que el discurso político en España constituye el sujeto político "madre», reconociendo y privilegiando a determinadas mujeres-madres e invisibilizando a otras, que quedan fuera del marco de reconocibilidad de lo maternal. Tanto la constitución política de la maternidad como de la madre misma sirven, en último término, para legitimar un modelo de feminidad y familia ligado a la pauta heteronormativa de comportamiento.

En este punto y, a modo de conclusión, regresaremos a las tres hipótesis planteadas al comienzo de esta investigación con el fin de subrayar la aportación que aspira a realizar este trabajo en el ámbito del análisis postestructural de políticas públicas. La primera hipótesis afirmaba que el análisis del discurso de nuestras políticas importa en la medida en que tiene un impacto en la vida cotidiana de las personas y, particularmente, porque es productor de espacios de vulnerabilidad. En concreto, el foco de este trabajo se sitúa sobre un tipo de vulnerabilidad específica: una vulnerabilidad de los cuerpos políticamente inducida. En el caso de estudio de la regulación de la interrupción del embarazo, se ha mostrado cómo las normas que se articulan a partir de un sistema de supuestos (la reforma del Código Penal de 1985 y el fallido Anteproyecto de 2013) tienden a producir o consolidar en mayor medida situaciones de vulnerabilidad corporal: la indemnidad de los cuerpos de las mujeres se pone en riesgo en la medida en que se veta diferencialmente una interrupción del embarazo segura en los servicios públicos de salud. Al mismo tiempo, la fórmula de los supuestos entiende que la mujer puede interrumpir su embarazo si los supuestos contemplados se lo permiten y no como el ejercicio de un derecho sobre su salud sexual y reproductiva, ni sobre el momento de la maternidad.

La segunda hipótesis sostenía que la manera en que se ha problematizado la maternidad en parte del discurso político en España ha tendido a producir o reforzar jerarquías entre las propias mujeres, y a consolidar una pauta heteronormativa de feminidad y familia. En lo que se refiere a la regulación de la posibilidad de interrupción del embarazo, se ha mostrado cómo los sistemas de supuestos pueden situar a mujeres que desean interrumpir un embarazo (y que no pueden acogerse a los supuestos planteados en la norma) en un espacio de vulnerabilidad, si además no pueden acudir a la sanidad privada para interrumpir su gestación de manera segura. Y, con ello, se refuerzan no solo espacios de mayor vulnerabilidad para determinadas mujeres, sino también jerarquías entre mujeres que pueden (o no) acceder a espacios médicos seguros para la interrupción de un embarazo. También se ha mostrado en esta investigación que la regulación en el acceso a la reproducción asistida en la sanidad pública, en la medida en que solo permite acceder a este servicio a parejas en las que exista una patología médica, está de facto expulsando a mujeres en parejas lesbianas o mujeres sin pareja de la posibilidad de acogerse a este servicio. Con ello, se están constituyendo determinados cuerpos de mujer como cuerpos ajenos a los criterios de reconocibilidad de lo maternal. Y ello está proyectando una normatividad que refuerza la pauta heteronormativa de comportamiento. Con ello, se muestra cómo el 
discurso político sobre quién puede ser madre sirve para promover modelos determinados de feminidad y familia.

La tercera hipótesis se refería a cómo el discurso político produce sujetos políticos, es decir, explora el vínculo entre discursividad política y producción de subjetividad. En concreto, este trabajo de investigación ha mostrado la manera en que el discurso político produce un modelo de madre normativa que convierte a determinadas mujeres en madres imposibles. Desde esta perspectiva, el foco de análisis no se sitúa simplemente en los procesos de inclusión y exclusión que las normas producen, sino en un análisis de las vidas que se consideran significativas. Es decir, el interés del análisis no se centra tanto en (re)diseñar normas para que tengan un carácter más, sino en pensar de nuevo cómo las normas han de ser reformuladas, de manera que haya vidas que no queden fuera de un marco de reconocibilidad de derechos. Y ello atiende al fin, como señala Butler, de diseñar y aprobar normas que produzcan resultados más radicalmente democráticos (2010: 20).

Con todo ello, este artículo ha pretendido contribuir al análisis de políticas públicas desde el enfoque postestructural, una aproximación aún poco frecuente en la literatura en castellano de esta área de análisis. También se han abordado cuestiones como la manera en que el discurso político importa en la medida en que condiciona la vida de las personas afectadas por la norma y, especialmente, condiciona espacios diferenciales de vulnerabilidad de los cuerpos. Además, se ha explorado asimismo el discurso político como productor de ontología, visibilizando no solo los procesos de exclusión de la norma sino, más allá, cómo el discurso político y de nuestras políticas constituye diferencialmente vidas significativas. Todo ello se ha estudiado en debates actuales clave en los estudios de género, como son los relativos a la salud sexual y reproductiva, el entendimiento de la maternidad en el debate público y, por extensión, los debates sobre los modelos de feminidad y familia deseables; aspectos cruciales en debates sobre el cuerpo, que están alcanzando dominancia en nuestra esfera pública, como pueden ser los relativos a la gestación subrogada.

\section{AGRADECIMIENTOS}

Deseo agradecer a los/as evaluadores/as de la Revista Española de Ciencia Politica su tiempo y valiosas contribuciones. Agradezco, asimismo, la labor de los editores de la revista y de su secretaria de redacción. También deseo agradecer de manera especial a Elvira Burgos su lectura y recomendaciones en una versión previa de este texto.

\section{Referencias}

Albet, Abel y Núria Benach. 2012. Doreen Massey. Un sentido global del lugar. Barcelona: Icaria.

Artiaga, Alba. 2015. Producción política de los cuidados y de la dependencia: políticas públicas y organización social de los cuidados [tesis doctoral]. Serrano Pascual, Amparo y 
Carlos Prieto Rodríguez (dirs.), Universidad Complutense de Madrid, Madrid. Disponible en: http://eprints.ucm.es/32816/.

Bacchi, Carol L. 1996. The politics of affirmative action. "Women», equality and category politics. London: Sage.

Bacchi, Carol L. 1999. Women, policy and politics. The construction of policy problems. London: SAGE.

Bacchi, Carol L. 2009. Analysing policy. What's the problem represented to be? NSW: Pearson.

Bacchi, Carol L. 2012. «Why study problematizations? Making politics visible», Open Journal of Political Science, 2 (1): 1-8. Disponible en: https://doi.org/10.4236/ ojps.2012.21001.

Bacchi, Carol L. y Jennifer Bonham. 2014. «Reclaiming discursive practices as an analytic focus: Political implications», Foucault Studies, 17, 173-192. Disponible en: https://doi.org/10.22439/fs.v0i17.4298.

Bacchi, Carol L. y Susan Goodwin. 2016. Poststructural policy analisis. A guide to practice. Palgrave Pivot. Disponible en: https://doi.org/10.1057/978-1-137-52546-8.

Bacchi, Carol L. 2017. «Policies as gendering practices: Re-Viewing categorical distinctions", Journal of Women, Politics and Policy. 38, 1-22. Disponible en: https:// doi.org/10.1080/1554477X.2016.1198207.

Bletsas, Angelique. 2012. «Spaces between: Elaborating the theoretical underpinnings of the 'WPR' approach and its significance for contemporary scholarship", en Angelique Bletsas y Chris Beasley (eds.), Engaging with Carol Bacchi. Strategic interventions and exchanges. Adelaide: University of Aelaide Press.

Borrás, Víctor (ed.) 2014. Familias también: diversidad familiar, familias homoparentales (relatos de vida). Barcelona: Bellaterra.

Burgos, Elvira. 2008. Qué cuenta como una vida. La pregunta por la libertad en Judith Butler. Madrid: Antonio Machado Libros.

Burgos, Elvira. 2012. «Deconstrucción y subversión», en Patricia Soley-Beltrán y Leticia Sabsay (eds.), Judith Butler en disputa. Lecturas sobre la performatividad. Madrid: EGALES.

Burgos, Elvira. 2013. «Luchas por la libertad: cuerpos en acción», THÉMATA. Revista de Filosofia, 48: 203-216. Disponible en: https://doi.org/10.12795/themata.2013. i48.18.

Butler, Judith. 2002. Cuerpos que importan. Buenos Aires: Paidós.

Butler, Judith. 2003. "Violence, mourning, politics», Studies in Gender and Sexuality, 4 (1): 9-37. Disponible en: https://doi.org/10.1080/15240650409349213.

Butler, Judith. 2006a. Vida precaria. El poder del duelo y la violencia. Buenos Aires: Paidós.

Butler, Judith. 2006b. «E El parentesco es siempre heterosexual de antemano?», en Deshacer el género. Barcelona: Paidós.

Butler, Judith. 2009. "Subjects of Sex/Gender/Desire», en Ann Phillips (ed.), Feminism and Politics. New York: Oxford University Press.

Butler, Judith. 2010. Marcos de guerra. Las vidas lloradas. Barcelona: Paidós. 
Crenshaw, Kimberlé, W. 1991. "Mapping the margins: Intersectionality, identity politics, and violence against women of color", Stanford Law Review, 43 (6): 12411299. Disponible en: https://doi.org/10.2307/1229039.

De Mauro Rucovsky, Martín A. 2015. Cuerpos en escena. Materialidady cuerpo sexuado en Judith Butler y Paul B. Preciado. Madrid: Editorial EGALES.

Goodwin, Susan. 2011. "Analysing policy as discourse: Methodological advances in policy analysis», en Lina Markauskaite, Peter Freebody y Jude Irwin (eds.), Methodological choice and design. Scholarship, policy and practice in social and educational research. New York: Springer.

Goodwin, Susan. 2012. "Women, policy and politics. Recasting policy studies», en Angelique Bletsas y Chris Beasley (eds.), Engaging with Carol Bacchi. Adelaide: University of Adelaide Press.

Gras Velázquez, Adrián (coord.). 2014. "Todo sobre mi familia. Perspectivas de género». Revista Feminismo/s, 23 (núm. Monográfico).

Guba, Egon G. (ed.). 1990. The paradigm dialogue. Newbury Park: SAGE.

Hobson, Barbara, Jane Lewis y Birte Siim. 2002. Contested concepts in gender and social politics. Cheltenham Glos: Edward Elgar Publishing. Disponible en: https://doi. org/10.4337/9781781950340.

López Rodríguez, Silvia. 2015. "Relatos que condicionan experiencias: implicaciones de los relatos de las políticas públicas sobre violencia contra las mujeres y aborto en España», Revista de Estudios Políticos, 167: 165-191.

López Rodríguez, Silvia. 2016. «Políticas públicas y distribución de vulnerabilidad: el caso del discurso político sobre el aborto en España», en Maria Caterina La Barbera y Marta Cruells (coords.), Igualdad de género y no discriminación en España: evolución, problemas, perspectivas. Madrid: Centro de Estudios Políticos y Constitucionales.

Llopis, María. 2015. Maternidades subversivas. Tafalla: Txalaparta.

Massey, Doreen. 1993. "Power-geometry and a progressive sense of place», en Jon Bird, Barry Curtis, Tim Putnam, George Robertson y Lisa Tickner (eds.), Mapping the Futures. Local Cultures, Global Changes. London: Rouledge.

Massey, Doreen. 1994. Space, place and gender. Cambridge: Cambridge Polity Press. Massey, Doreen. 2008. For space. London: SAGE.

Massey, Doreen. 2012. «Espacio, lugar y política en la coyuntura actual», Urban, 4: 7-12.

Peterson, Elin. 2009. «Género y Estado del bienestar en las políticas españolas», Asparkia, 20: 35-57.

Peterson, Elin. 2015. «Framing caregiving work for older people in Spanish public policy: gender, power and social justice», Revista Española de Ciencia Política, 39: 221-237.

Spade, Dean. 2015. Una vida normal. Violencia administrativa, politicas trans criticas $y$ los limites del derecho. Barcelona: Bellaterra. Disponible en: https://doi. org/10.1215/9780822374794.

Squires, Judith. 1999. Gender in Political Theory. Cambridge: Polity Press. 
Staunaes, Dorthe. 2003. «Where have all the subjects gone? Bringing together the concepts of intersectionality and subjectification», NORA, Nordic Journal of Feminist and Gender Research, 11 (2): 101-110. Disponible en: https://doi. org/10.1080/08038740310002950.

Tanesini, Alessandra. 1994. «Whose language?», en Kathleen Lennon y Margaret Whitford (eds.), Knowing the difference. Feminist perspectives in epistemology. New York: Rouledge. Disponible en: https://doi.org/10.4324/9780203216125_chapter_13.

Trujillo, Gracia. 2016. «Mi cuerpo es mío. Parentalidades y reproducción no heterosexuales y sus conexiones con otras demandas», Viento Sur, 146: 61-68.

Verloo, Mieke y Emanuela Lombardo. 2007. «Contested gender equality and policy variety in Europe: Introducing a critical frame analysis approach", en Mieke Verloo (ed.), Multiple meanings of gender equality. A critical frame analysis of gender policies in Europe. Budapest: CEU Press.

Warner, Michael. 1993. Fear of a queer planet: Queer politics and social Theory. Minneapolis: University of Minnesota Press.

Wittig, Monique. 2005 [1992]. El pensamiento heterosexual. Madrid: Egales.

Young, Iris M. 2005. On female body experience. New York: Oxford University Press. Disponible en: https://doi.org/10.1093/0195161920.001.0001.

Presentado para evaluación: 22 de agosto de 2016.

Aceptado para publicación: 13 de junio de 2017.

\section{SILVIA LÓPEZ-RODRÍGUEZ}

email

Investigadora en el ámbito de las políticas de igualdad de género. Sus intereses de investigación se relacionan con la teoría feminista contemporánea sobre el cuerpo, las políticas sobre violencias de género y los derechos sexuales y reproductivos. Ha sido profesora ayudante en la Universidad Autónoma de Madrid, donde en los próximos meses defenderá su tesis doctoral. 